\title{
Corrigendum
}

\section{Obatoclax (GX15-070) triggers necroptosis by promoting the assembly of the necrosome on autophagosomal membranes}

\author{
F Basit, S Cristofanon and S Fulda
}

Cell Death and Differentiation (2014) 21, 1183-1184; doi:10.1038/cdd.2014.52

Correction to: Cell Death and Differentiation (2013) 20, 1161-1173; doi:10.1038/cdd.2013.45; published online 7 June 2013

Since the publication of this article, the authors have noticed that some blank blots were unintentionally mixed up in Figures 5 and 6.

a

TE671

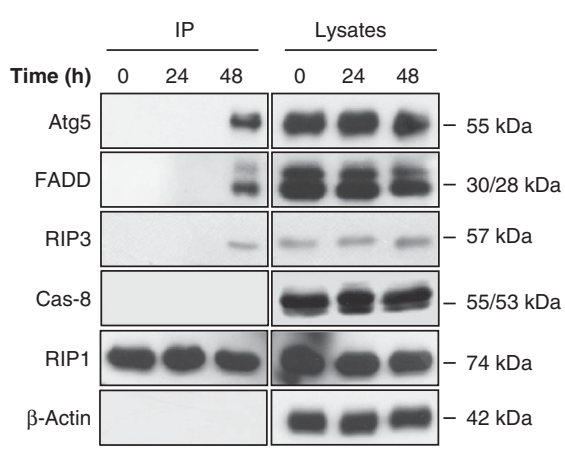

b

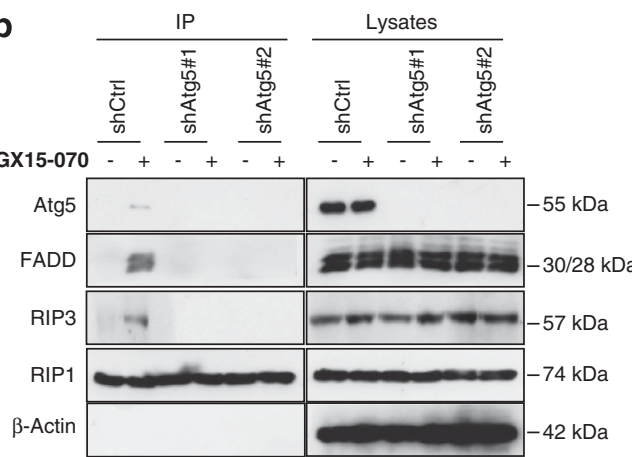

The corrected panels of Figures $5 \mathrm{a}, \mathrm{b}$ and $6 \mathrm{e}$ appear below.

Although this unintentional mistake does not alter the findings or the conclusions drawn in the original published study, the authors want to clarify the issue and apologize for the error.
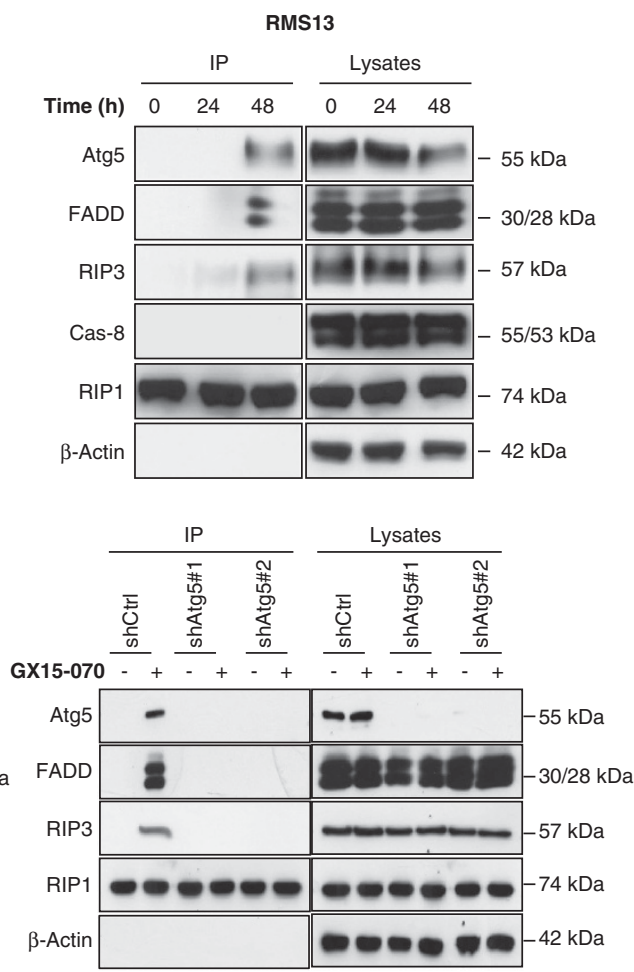

Figure 5 GX15-070 triggers the assembly of the necrosome on autophagosomes. (a) TE671 and RMS13 cells were treated with $200 \mathrm{nM}$ GX15-070 for the indicated time points. RIP1 was immunoprecipitated (IP) using anti-RIP1 antibody. Atg5, FADD, RIP3 and caspase-8 were detected by western blotting. (b) TE671 and RMS13 cells were transduced with control vector (shCtrl) or vectors containing two distinct shRNA sequences against Atg5 (shAtg5\#1, shAtg5\#2) and treated with 100 nM GX15-070 for 48 h. RIP1 was immunoprecipitated (IP) using anti-RIP1 antibody. Atg5, FADD, RIP3 and caspase-8 were detected by western blotting 

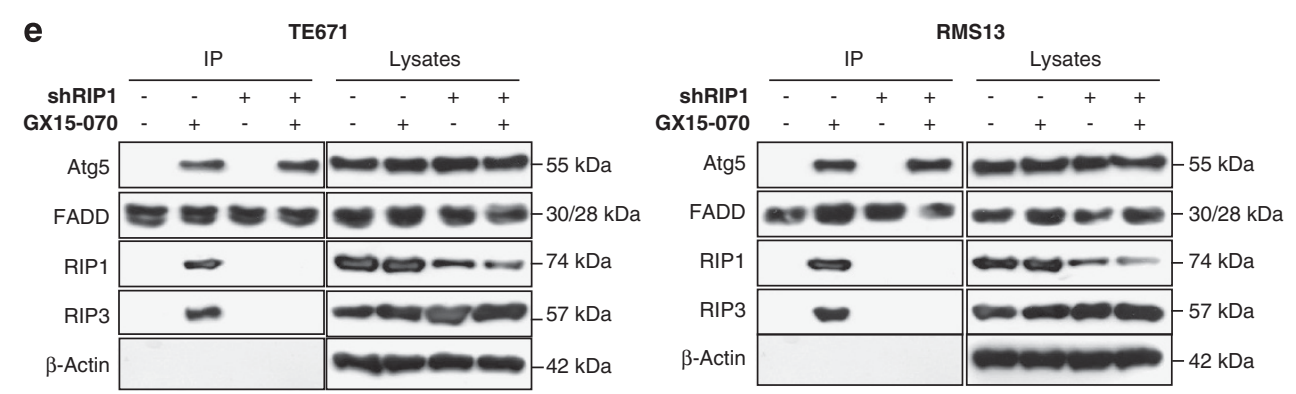

Figure 6 RIP1 is required for GX15-070-induced cell death downstream of autophagosome formation. (a) TE671 and RMS13 cells were treated with $200 \mathrm{nM}$ GX15-070 in the presence or absence of $40 \mathrm{mg} / \mathrm{ml} \mathrm{Nec-1}$. Cell viability was determined by MTT assay and is expressed as percentage of untreated controls. (b) TE671 and RMS13 cells were transduced with control vector (shCtrl) and vector containing shRNA sequence against RIP1 (shRIP1). Expression of RIP1 was determined by western blotting. (c) TE671 and RMS13 cells were treated with the indicated concentrations of GX15-070 for $72 \mathrm{~h}$. Cell viability was determined by MTT assay and is expressed as percentage of untreated controls. (d) TE671 and RMS13 cells were treated with $100 \mathrm{nM} \mathrm{GX15-070} \mathrm{for} 72 \mathrm{~h}$ before medium was exchanged by fresh drug-free medium. Colonies were stained with crystal violet and counted under the microscope. The percentage of colony numbers in the presence and absence of GX15-070 is shown. (e) TE671 and RMS13 cells were treated with $100 \mathrm{nM}$ GX15-070 for $48 \mathrm{~h}$. FADD was immunoprecipitated (IP) using anti-FADD antibody. Atg5, FADD, RIP1 and RIP3 were detected by western blotting. (f) TE671 and RMS13 cells were treated with $100 \mathrm{nM}$ GX15-070 for the indicated time points and LC3 lipidation was detected by western blotting. In panels a, $\mathbf{c}$ and d, data represent mean + S.D. of three independent experiments performed in triplicate; ${ }^{*} P<0.05 ;{ }^{* *} P<0.001$ comparing control with RIP1 knockdown cells (c and d) or cells treated in the presence and absence of Nec-1 (a) 